\title{
Secretory breast carcinomas with ETV6-NTRK3 fusion gene belong to the basal-like carcinoma spectrum
}

\author{
Marick Laé, Paul Fréneaux, Xavier Sastre-Garau, Olfa Chouchane, Brigitte Sigal-Zafrani \\ and Anne Vincent-Salomon
}

Service de Pathologie, Section Médicale, Institut Curie, Paris Cedex, France

\begin{abstract}
Secretory breast carcinomas ( $<\mathbf{0 . 1 5} \%$ of breast tumors) are associated with a characteristic morphology and a favorable prognosis. Remarkably, this entity is the only epithelial tumor of the breast with a balanced translocation, $t(12 ; 15)$, that creates an ETV6-NTRK3 gene fusion encoding chimeric tyrosine kinase also encountered in cellular mesoblastic nephroma and infantile fibrosarcoma. The aim of this study was to determine the phenotypic class (ie luminal A/B, ERBB2, basal-like) of secretory breast carcinoma. A series of six secretory breast carcinomas were identified in our files. The ETV6 rearrangement was confirmed in all cases by fluorescence in situ hybridization. Immunophenotype was assessed with anti-ER, PR, ERBB2, KIT, EGFR, E-cadherin, vimentin, PS100, smooth muscle actin, basal (CK5/6 and 14), luminal cytokeratins (CK8/18) and p63 antibodies. In situ and invasive components shared the same immunoprofile and were ER, PR, ERBB2 negative with expression of basal cytokeratins. ETV6 gene alterations were present in both in situ and invasive components, highlighting their genetic similarities. The immunoprofile data (triple-negative with expression of basal markers) showed that secretory breast carcinomas with ETV6-NTRK3 fusion gene belong to the phenotypic basal-like spectrum of breast carcinomas. These results support the hypothesis that secretory breast carcinomas have immunohistochemical and genetic features that distinguish them from other basal-like tumors of the breast.
\end{abstract}

Modern Pathology (2009) 22, 291-298; doi:10.1038/modpathol.2008.184; published online 14 November 2008

Keywords: Secretory breast carcinoma; basal-like carcinoma; breast; ETV6-NTRK3; translocation; basal cytokeratins

Secretory breast carcinoma is one of the rarest types of breast cancer, accounting for less than $0.15 \%$ of all breast cancers. ${ }^{1}$ This entity was initially termed 'Juvenile breast carcinoma' by McDivitt and Stewart, ${ }^{2}$ as the average age of the seven patients in their series was 9 years, with a range of 3-15 years. More cases in children and adults have subsequently been described. ${ }^{3,4}$ The age at presentation ranges from 3 to 87 years with a median of 25 years. ${ }^{1}$ It was therefore recommended to replace the term "juvenile breast carcinoma' by the descriptive term 'secretory breast carcinoma'.

Secretory breast carcinomas are distinguished from invasive ductal carcinomas of the breast by

Correspondence: Dr A Vincent-Salomon, Department of Pathology, Institut Curie-Hôpital, 26 rue d’Ulm, 75248 Paris Cedex 05, France.

E-mail: anne.salomon@curie.net

Presented in part at the Annual United States and Canadian Academy of Pathology, Denver, 2008.

Received 15 April 2008; revised and accepted 23 June 2008; published online 14 November 2008 their characteristic histomorphology. Secretory breast carcinomas can demonstrate several histologic patterns including, solid, microcystic and tubular, and many tumors contain all three patterns. Tumor cells are polygonal with granular eosinophilic cytoplasm. Atypia is minimal or absent and mitotic activity is low. A typical finding is the presence of intracellular and extracellular secretory material (periodic acid-Schiff and Alcian blue positive).

Tognon et $a l^{5}$ recently showed that secretory breast carcinomas are associated with a characteristic balanced translocation, $\mathrm{t}(12 ; 15)$, that creates a ETV6-NTRK3 gene fusion. The fusion between ETV6 (TEL) transcription factor and the NTRK3neurotrophin-3 tyrosine kinase protein occurs in various cell types and lineages. ${ }^{5}$ This specific translocation was first cloned in pediatric mesenchymal cancers: congenital fibrosarcoma and congenital cellular mesoblastic nephroma, two morphologically similar pediatric mesenchymal tumors with no epithelial features. ${ }^{6,7}$ The biological 
consequence of this translocation is the expression of a chimeric tyrosine kinase protein with a potent transforming activity in very different cell lineages including fibroblasts, and breast epithelial cells. ${ }^{8}$ The finding of a fusion transcript in secretory breast carcinomas and the demonstration that ETV6NTRK3 can transform normal murine mammary epithelial cells strongly suggest that this chimeric protein constitutes a primary genetic lesion in this subtype of breast carcinoma. ${ }^{8}$ The ETV6-NTRK3 fusion activates RAS-MAP kinases and PI3K-Akt pathways which are important for breast cell proliferation and survival. ${ }^{8}$

Gene expression profile analysis has recently identified at least six different breast carcinoma types: luminal A, B, C, ERBB2, basal-like and normal-like types. ${ }^{9,10}$ Subsequent immunophenotype analyses provided surrogate markers to identify these molecular types in clinical practice. ${ }^{11,12}$

Secretory breast carcinoma is a paradoxical entity harboring a translocation known to be oncogenic in two other tumor types, including a mesenchymal tumors, while also demonstrating true epithelial differentiation with secretory activity. In this context, the aim of this study was to identify the secretory breast carcinoma phenotypic class. Six histologically and molecularly confirmed secretory breast carcinomas were evaluated. Secretory breast carcinomas were found to be low-grade triple-negative (ER, PR and ERBB2 negative) carcinomas that express basal-cell markers. This observation suggests that secretory breast carcinomas with ETV6-NTRK3 fusion gene are part of the basal-like spectrum. ETV6 gene alterations were present in both the in situ and invasive components, highlighting their genetic similarities. These results also show that secretory breast carcinomas have genetic features that distinguish them from other basal-like tumors of the breast.

\section{Materials and methods}

\section{Tumor Samples}

A series of six tumors diagnosed as secretory breast carcinoma (six patients) was selected from the Institut Curie Department of Pathology files between 1990 and 2007. Tissue was obtained after tumorectomy or mastectomy. Histologic examination on hematoxylin-eosin-saffron-stained slides was performed by two of the authors (ML, AVS) to independently confirm the diagnosis. Consistent, precise and reproducible pathologic grading (differentiation, mitotic index, cytonuclear atypias) was performed according to the system of Elston and Ellis. The mitotic index was evaluated per 10 highpower fields (field diameter $0.61 \mathrm{~mm}$ ). Pathologic tumor size, peritumor vascular invasion, in situ component, perineural involvement, and axillary lymph node involvement were assessed.
Table 1 Clone, source and dilution of primary antibodies used for immunohistochemical analysis of secretory breast carcinomas

\begin{tabular}{llll}
\hline Antibody & Clone & Source & Dilution \\
\hline Cytokeratin 5/6 & D5/16B4 & Dako & $1 / 50$ \\
Cytokeratin 8/18 & DC10 & Zymed & $1 / 200$ \\
Smooth muscle actin & 1A4 & Dako & $1 / 400$ \\
p63 & 4 A4 & Dako & $1 / 100$ \\
Estrogen receptor & 6F11 & Novocastra & $1 / 50$ \\
Progesterone receptor & 1A6 & Novocastra & $1 / 200$ \\
ERBB2 & CB11 & Novocastra & $1 / 800$ \\
KIT (CD117) & Polyclonal & Dako & $1 / 200$ \\
Cytokeratin 14 & LL002 & Biogenex & $1 / 50$ \\
EGFR & 31G7 & Zymed & $1 / 40$ \\
Vimentin & 3B4 & Dako & $1 / 400$ \\
S100 & Polyclonal & Dako & $1 / 1600$ \\
E-cadherin & 4A2C7 & Zymed & $1 / 100$ \\
\hline
\end{tabular}

\section{Immunohistochemical Analyses}

Immunostaining was performed on $4 \mu \mathrm{m}$ tissue sections prepared from a representative sample of the tumor. After rehydration and antigen retrieval in citrate buffer ( $10 \mathrm{mM}, \mathrm{pH} 6.1)$, tissue sections were stained for estrogen receptor (ER), progesterone receptor (PR), ERBB2/neu, CD117 (an epitope of KIT), smooth muscle actin, E-cadherin, vimentin, EGFR, p63 and a panel of cytokeratins (CK5/6, CK14, luminal CK8/18). Source and dilution procedures are listed in Table 1. Staining was revealed with the Vectastain Elite ABC peroxidase mouse IgG kit (Vector, Burlingame, CA, USA) using diaminobenzidine (Dako A/S, Glostrup, Denmark) as chromogen. Positive nuclear staining for ER, PR and P63 was recorded according to standardized guidelines. For ERBB2, only membranous staining was interpreted, as previously defined. ${ }^{13}$ Expression of CD117, CK14, CK5/6, CK8/18 was scored positive when any cytoplasmic and/or membranous tumor cell staining was observed. The percentage of tumor cells with positive immunostaining for each antibody was assessed. Internal and external controls were included in the experiments for each antibody.

\section{Fluorescence In Situ Hybridization Analysis}

To identify the ETV6 rearrangement in breast cancer cells, interphase fluorescence in situ hybridization was performed with an ETV6 (TEL) split-apart dual color probe (Vysis; Abbott laboratories, IL, USA) on formalin-fixed, paraffin-embedded tumor tissue sections with both invasive and in situ components. Each $4-\mu \mathrm{m}$ section was deparaffinized in xylene and rehydrated in a $100-85-70 \%$ ethanol series, then washed in PBS. After proteolytic treatment for paraffin-embedded samples, the tissue section and probe were co-denaturated at $85^{\circ} \mathrm{C}$ for $5 \mathrm{~min}$ then hybridized at $37^{\circ} \mathrm{C}$ overnight (Thermobrite ${ }^{\mathrm{TM}}$ ). Probes were visualized as green and red. Nuclei were counterstained using a DAPI/antifade solution. Slides were viewed with a DAPI/rhodamine/fluorescein 
Table 2 Clinicopathologic data for six secretory breast carcinomas

\begin{tabular}{|c|c|c|c|c|c|c|}
\hline Case & Case 1 & Case 2 & Case 3 & Case 4 & Case 5 & Case 6 \\
\hline Age (yo) & 58 & 47 & 60 & 55 & 25 & 38 \\
\hline Sex & $\mathrm{F}$ & $\mathrm{F}$ & $\mathrm{F}$ & $\mathrm{F}$ & $\mathrm{F}$ & $\mathrm{F}$ \\
\hline Size (mm) & 11 & 15 & 40 & 20 & 15 & 5 \\
\hline GRADE (EE) & 1 & 2 & 2 & 2 & 2 & 1 \\
\hline Differentiation & 1 & 2 & 2 & 2 & 2 & 2 \\
\hline Atypias & 3 & 3 & 3 & 3 & 3 & 2 \\
\hline Number of mitosis/10 HPF & 0 & 8 & 2 & 2 & 2 & 0 \\
\hline Vascular invasion & 0 & 0 & 0 & 0 & Yes & 0 \\
\hline In situ component (\%) & 30 & 40 & 10 & 40 & 20 & 5 \\
\hline In situ component nuclear grade & Intermediate & High with necrosis & Intermediate & Intermediate & Intermediate & Intermediate \\
\hline $\begin{array}{l}\text { Pathologic axillary lymph node } \\
\text { status }\end{array}$ & $\begin{array}{l}0 / 5 \text { sentinel } \\
\text { lymph nodes }\end{array}$ & $0 / 6 \mathrm{~N}$ & NA & NA & $2 \mathrm{~N}+/ 13 \mathrm{~N}$ & $\begin{array}{l}0 / 1 \text { sentinel } \\
\text { lymph node }\end{array}$ \\
\hline Stage & T1NOMO & T1NOMO & $\mathrm{T} 2 \mathrm{NxMx}$ & $\mathrm{T} 2 \mathrm{NxMx}$ & T1N1MO & T1NOMO \\
\hline Treatment & S RT & S RT & NA & NA & S CT RT & $\mathrm{S}$ \\
\hline Follow-up (months) & NOD (16 m) & NOD $(78 \mathrm{~m})$ & NA & NA & NOD $(84 \mathrm{~m})$ & NOD (2 m) \\
\hline
\end{tabular}

CT, chemotherapy; EE, Elston Ellis; HPF, high-power field; NA, not available; NOD, no evidence of disease; RT, radiotherapy; S, surgery; yo, years old.

filter, and images were captured with a CDD camera, filtered and processed with Applied Imaging system using a Leica Microsystems microscope. At least 50 interphase nuclei were analyzed per hybridization.

\section{Results}

\section{Clinical Features}

All patients were women between the ages of 25 and 60 years with a mean age of 47 years. The most frequent clinical presentation was an asymptomatic mobile mass or radiologic lesion. At the time of diagnosis, none of the patients presented suspicious axillary lymph nodes or metastatic disease. All six patients underwent primary surgery (lumpectomy). Further treatment details and follow-up were available for four patients (two were consultation cases with no available follow-up). Axillary lymph node dissection was performed in two patients and sentinel lymph node dissection was performed in two patients. Three (75\%) of the patients with known lymph node status were lymph node metastasis negative, whereas one was pN1 (one positive node). Three patients received adjuvant radiotherapy. The patient with lymph node metastasis received adjuvant chemotherapy. All four patients were alive with no evidence of recurrence after treatment. Median clinical follow-up was 45 months (range: 2-84 months). Genetic analysis performed on the patient who presented a tumor at the age of 25 years did not show any BRCA1 and BRCA2 alteration. Table 2 summarizes the main clinical features of this series of cases of secretory breast carcinoma.

\section{Pathologic Findings}

Tumor size ranged from 5 to $40 \mathrm{~mm}$, with a mean of $17 \mathrm{~mm}$. Microscopically, all tumors presented both invasive and in situ components. Tumors demon- strated several histologic patterns including, solid, microcystic, and tubular, and many tumors contained all three patterns. Tumor cells were polygonal with granular eosinophilic cytoplasm. Atypia was moderate to marked. Nuclei were ovoid with small nucleoli. Mitotic activity was zero or low (mitotic activity ranged from 0 to $8 / 10 \mathrm{HPF}$ ). A typical finding was the presence of intracellular (intracytoplasmic lumina) and extracellular secretory material (PAS and Alcian blue positive). Four tumors were Elston and Ellis grade $2(66 \%)$ and two were grade 1 $(33 \%)$. Vascular invasion was present in one case. Perineural infiltration was absent in all cases. Necrosis was absent in the invasive component. All tumors presented an in situ secretory component with cribriform, microcystic or massive architecture at the margins or within the tumor. In situ nuclear grade was intermediate without necrosis except for one tumor which presented a high-grade in situ component with necrosis. Pathologic findings are reported in Table 2 and illustrated in Figure 1a-c.

\section{Immunohistochemical Findings}

Immunohistochemical findings are reported in Table 3 and illustrated in Figure 1d-i. All tumors were negative for ER, PR and ERBB2. All tumors expressed CK8/18 at least focally (1-80\% of cells). E-cadherin expression (membranous immunoreactivity of $5-90 \%$ of cells) was present in all tumors. Five tumors (83\%) expressed CK5/6 (1-80\% of cells), vimentin (5-60\% of cells). Four tumors focally expressed KIT (1-5\% of cells). CK14 expression was present in two tumors and EGFR expression was present in three tumors. Five tumors (83\%) expressed S-100 (strong and diffuse expression in $100 \%$ of cells) with focal smooth muscle actin expression $(2-10 \%$ of cells). None of the tumors cells expressed p63. In situ and invasive components presented the same immunoprofile. 

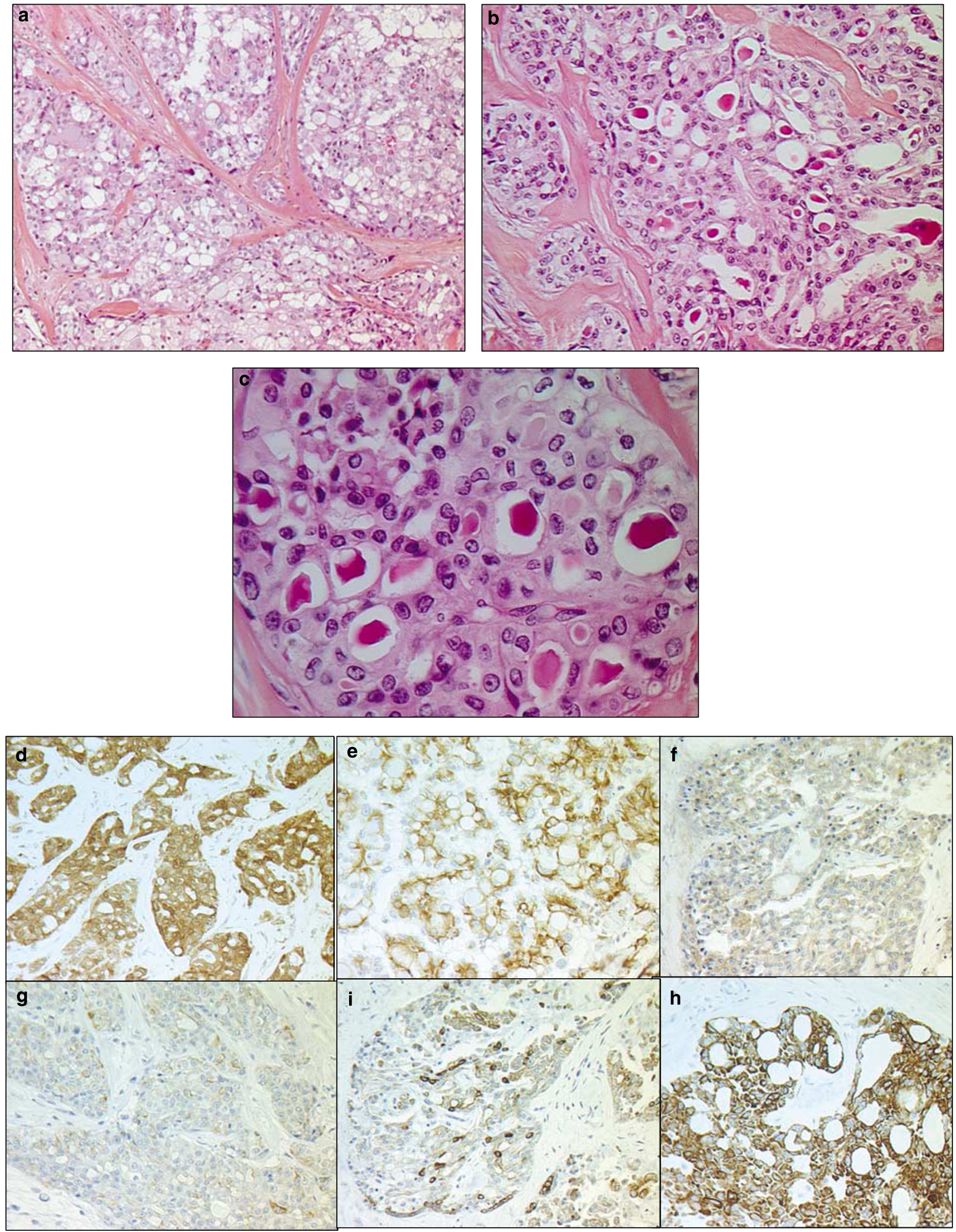

Figure 1 Example of characteristic histologic pattern and immunophenotype of a juvenile secretory breast carcinoma. Secretory breast carcinoma with microacinar pattern (hematoxylin-eosin-saffron stains-HES, original magnification $\times 100$, a). Tumor cells with abundant intracellular and extracellular secretory material (HES, original magnification $\times 200$, b). Tumor cells with finely vacuolated cytoplasm and nuclei with nucleoli (HES, original magnification $\times 400$, c). Immunohistochemical staining for S-100 (d), vimentin (e), EGFR (f), for CK5/6 (g), CK14 (i) and CK8/18 (h). 
Table 3 Immunophenotype and FISH results of six secretory breast carcinomas

\begin{tabular}{|c|c|c|c|c|c|c|}
\hline Case & Case 1 & Case 2 & Case 3 & Case 4 & Case 5 & Case 6 \\
\hline ER & $5 \%$ weak & $0 \%$ & $0 \%$ & $0 \%$ & $0 \%$ & $0 \%$ \\
\hline PR & $0 \%$ & $0 \%$ & $0 \%$ & $0 \%$ & $0 \%$ & $0 \%$ \\
\hline HER2 & $0 \%$ & $0 \%$ & $0 \%$ & $0 \%$ & $0 \%$ & $0 \%$ \\
\hline CK8/18 & $10 \%$ & $1 \%$ & $4 \%$ & $4 \%$ & $5 \%$ & $80 \%$ \\
\hline CK5/6 & $1 \%$ & $40 \%$ & $80 \%$ & $0 \%$ & $60 \%$ & $2 \%$ \\
\hline CK14 & $0 \%$ & $90 \%$ & $0 \%$ & $0 \%$ & $1 \%$ & $0 \%$ \\
\hline p63 & $0 \%$ & $0 \%$ & $0 \%$ & $0 \%$ & $0 \%$ & $0 \%$ \\
\hline SMA & $2 \%$ & $10 \%$ & $3 \%$ & $3 \%$ & $2 \%$ & $0 \%$ \\
\hline S100 & $100 \%$ & $100 \%$ & $0 \%$ & $100 \%$ & $100 \%$ & $100 \%$ \\
\hline Vimentin & $0 \%$ & $5 \%$ & $10 \%$ & $60 \%$ & $30 \%$ & $5 \%$ \\
\hline KIT & $3 \%$ & $0 \%$ & $0 \%$ & $1 \%$ & $5 \%$ & $1 \%$ \\
\hline EGFR & $100 \%$ moderate & $100 \%$ moderate & $0 \%$ & $0 \%$ & $0 \%$ & $15 \%$ moderate \\
\hline E-cadherin & $90 \%$ weak & $90 \%$ weak & $60 \%$ & $5 \%$ & $25 \%$ weak & $70 \%$ moderate \\
\hline ETV6 rearrangement & Present & Present & Present & Present & Present & Present \\
\hline
\end{tabular}

RE, estrogens receptors; RP, progesterone receptors; SMA, smooth muscle actin.
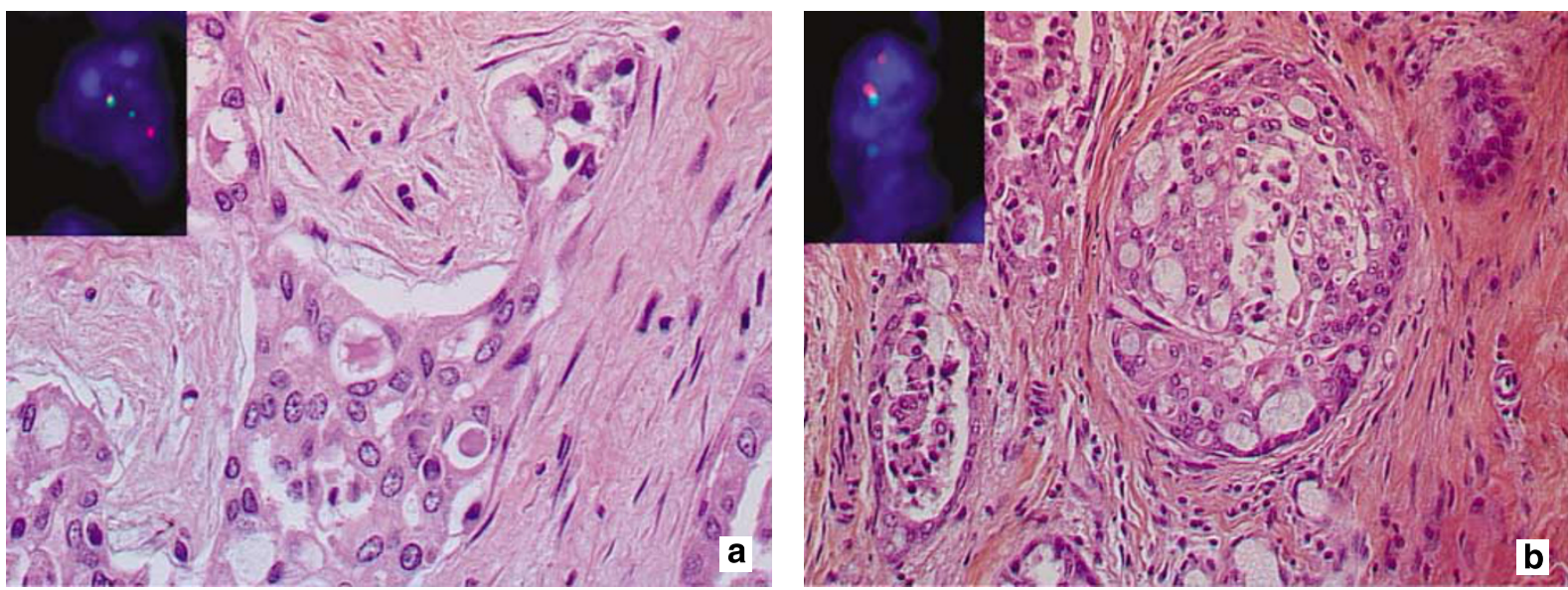

Figure 2 Dual color interphase FISH on paraffin-embedded tumor tissue sections of an invasive secretory breast carcinoma (a) and its in situ component (b). FISH images illustrate the presence of a rearrangement of the ETV6 gene. Split of the FITC-labeled (green) and rhodamine-labeled (red) signals flanking the ETV6 locus on chromosome 12. Intact copies of the ETV6 regions are seen as pairs of adjacent green and red signals.

\section{Molecular Features}

All six secretory breast carcinomas had adequately preserved nuclei for fluorescence in situ hybridization analysis. Interphase fluorescence in situ hybridization with a split ETV6 probe showed split signals in epithelial cells (Figure 2a) in both the invasive and in situ components. Normal breast tissue showed fused signals (internal control). Fluorescence in situ hybridization results are reported in Table 3 and illustrated in Figure 2. The results of histologic examination and fluorescence in situ hybridization assays with a split apart ETV6 probe were concordant in every case.

\section{Discussion}

In our experience, secretory breast carcinomas are rare tumors, representing less than $0.15 \%$ of all breast tumors. Six secretory breast carcinomas were identified in a series of 11900 newly diagnosed breast carcinomas at Institut Curie between 2000 and 2007, representing an incidence of $0.0005 \%$. These results are concordant with those of the literature: in a retrospective study of 7038 breast carcinoma cases, Lamovec and Bracko ${ }^{14}$ reported four cases of SBC and Botta et a ${ }^{15}$ found one case of secretory carcinoma in a series of 3000 breast carcinoma cases.

This study concerns a series of six molecularly confirmed secretory breast carcinoma. As previously described, this tumor has a very specific histologic pattern (cystic, microcystic, glandular, solid, tubular), abundant intracellular and extracellular secretion material containing mucin and a vacuolated or granular eosinophilic cytoplasm. Elston and Ellis grading in this series was 1 or 2 . Atypia was minimal or moderate and mitotic activity was low. This tumor was frequently associated with an in situ component. All tumors expressed CK8/18 and 
E-cadherin. This immunophenotype confirmed the epithelial differentiation of this tumor initiated by an oncogenic event, the $t(12 ; 15)$ translocation also encountered in mesenchymal tumors such as infantile fibrosarcoma. All secretory breast carcinomas studied demonstrated a triple negativity for ER/PR and ERBB2 associated with either CK5/6 and/or CK14 and/or KIT expression. Secretory breast carcinomas were therefore shown to present a basal-like phenotype, as defined by Nielsen et al. ${ }^{11}$ P63 (a myoepithelial marker) was not expressed. The in situ and invasive components shared the same immunoprofile. All secretory breast carcinomas showed PS100 and smooth muscle actin expressions. These results support the hypothesis that secretory breast carcinomas have immunohistochemical features that distinguish them from other types of invasive breast carcinomas. Immunohistochemical analysis using the combination of these markers (CK5/6, CK8/18, S100, smooth muscle actin and E-cadherin) could be helpful in difficult histologic cases.

Basal-like breast cancers initially identified from gene expression profile analyses are a distinct group of tumors with several morphologic features (grade 3 , pushing border, lymphoid stroma and central fibrosis/necrosis are the dominant morphologic findings). 'Basal-like' breast cancers defined by gene expression profile analyses have been associated with a significantly poorer overall and disease-free survival compared to most other breast carcinoma subclasses. ${ }^{10}$ As described by Nielsen et $a l^{11}$ and others, ${ }^{12}$ a panel of four antibodies (ER, HER1/ EGFR, ERBB2 and cytokeratin 5/6) can accurately identify basal-like tumors in clinical practice, KIT expression is also useful. ${ }^{11}$ Although most triplenegative tumors with a basal-like immunophenotype are presumed to be high-grade tumors (medullary carcinoma, ${ }^{16}$ metaplastic carcinoma $^{17}$ and invasive ductal carcinoma, a small subset of invasive cancers of special types such as adenoid cystic carcinomas have also been shown to present a basal-like immunophenotype. ${ }^{3,18}$ Assessment of a basal-like immunophenotype in clinical practice further demonstrated that the basal-like spectrum of tumors encompasses various histologic types of tumors associated with different prognoses. The present immunoprofile results show that secretory breast carcinomas are triple-negative and express basal markers. The immunophenotype of this entity therefore overlaps with that of basal-like breast carcinomas. These findings also illustrate the heterogeneity of phenotypically defined basal-like carcinomas. In contrast to other so-called basal-like breast carcinomas and like adenoid cystic carcinoma, secretory breast carcinomas have a favorable prognosis, at least in this small series. These observations emphasize the need for a definition of basal-like tumors based not only on immunophenotypic characteristics but also on morphologic parameters such as high histologic grade and high level of proliferation. It also indicates the need to identify other specific markers of basal-like/highgrade carcinomas.

Notably, in contrast with other grade 3 basal-like carcinomas that are infrequently associated with in situ carcinoma, ${ }^{19}$ the secretory breast carcinomas in this series were always associated with an in situ component, constituting another important characteristic of this entity.

To date, recurrent gene fusions have been identified in a subgroup of epithelial malignancies (renal, prostate, thyroid, salivary gland and sinonasal carcinomas). However, ETV6-NTRK3 fusion protein is identified in mesenchymal and epithelial malignancies. Congenital cellular mesoblastic nephroma and infantile fibrosarcoma, two pediatric tumors expressing the ETV6-NTRK3 gene fusion, also demonstrate chromosome 11 trisomy. ${ }^{7}$ Fluorescence in situ hybridization is a useful ancillary diagnostic test for the detection of the ETV6 rearrangement. ETV6 rearrangement is restricted to secretory breast carcinomas as demonstrated by tissue microarray studies on a panel of 201 breast carcinomas $(100 \%$ specificity). ${ }^{20}$ Interestingly in our series, ETV6 gene alterations were present in both in situ and invasive components, highlighting their genetic similarity. Fluorescence in situ hybridization could be useful to rule out differential diagnoses such as apocrine carcinoma, lipid- or glycogen-rich lobular, ductal or mucinous carcinoma, acinic carcinoma, signet ring carcinoma, hypersecretory hyperplasia, lactating adenoma, lactating changes, collagen spherulosis and to identify secretory breast carcinomas among basal-like carcinomas sharing the same immunoprofile.

Surgery is considered to be the primary treatment for secretory breast carcinoma. However, due to of the very small number of reported cases, no guidelines for surgical management have been published. Conservative treatment should be conducted according to the same multidisciplinary guidelines as for other invasive breast carcinomas. The overall incidence of axillary lymph node involvement is about $30 \%$ in children and adults according to earlier studies and meta-analyses of case reports. ${ }^{21}$ Axillary lymph node dissection or sentinel node biopsy is therefore indicated in the presence of an invasive component. Postoperative radiotherapy and adjuvant chemotherapy have been used by several teams, but there is not sufficient evidence at the present time to recommend either approach in the management of secretory breast carcinoma.

Distant metastases from secretory breast carcinomas are extremely rare $^{21}$ with only four reported cases. ${ }^{22}$ Previous published series and case reports indicated that secretory breast carcinoma is associated with a more favorable outcome than other subtypes of invasive breast carcinomas. ${ }^{11}$ However, more recent studies have showed that outcome is age-related. ${ }^{23}$ Although only limited follow-up data were available, none of the patients of the present 
series died from their disease, or presented secondary metastatic relapse. However, there are many reported cases of patients who experienced local recurrence after a long disease-free interval, but these reports concern patients treated by conservative surgery. ${ }^{21}$ Metastatic secretory breast carcinomas are not chemosensitive, as all patients treated with chemotherapy showed disease progression while on treatment. These findings contrast with previous reports on the high chemosensitivity to common agents (vincristine, cyclophosphamide, adriamycin, dactinomycin and ifosfamide) of congenital fibrosarcomas and cellular mesoblastic nephromas, two other neoplasms associated with the ETV6-NTRK3 fusion gene. ${ }^{24}$ This suggests that secretory breast carcinomas, perhaps due to their slowly growing behavior, acquire additional genetic alterations that ultimately confer chemoresistance. It would be very useful to establish cancer cell lines from this tumor type and mouse models (as performed by $\mathrm{Li}$ et $a l^{25}$ ) to study the genetic pathogenesis, cellular origins and chemoresistance and to generate preclinical models. The fact that this breast carcinoma is associated with an ETV6-NTRK3 fusion gene highlights the function of this gene and its downstream signaling in breast cancer and also suggests a putative target for drug development.

In conclusion, secretory breast carcinomas are low-grade triple-negative carcinomas that belong to the basal-like spectrum and that harbor a translocation. In contrast with high-grade basal-like carcinomas, this tumor is frequently associated with an in situ component. ETV6 gene alterations are present in both in situ and invasive components, highlighting their genetic similarities. In situ and invasive component also share the same immunoprofile. These results support the hypothesis that secretory breast carcinomas have immunohistochemical and genetic features that distinguish them from other phenotypically defined basal-like/tumors of the breast.

\section{Acknowledgement}

We thank Marie-Jo Birolini and Frédérique Viard for technical assistance and Dr Hofmann-Guilaine, Dr Kadi-Hanifi, Champagne de Labriolle for providing pathology materials.

\section{References}

1 Tavassoli FA, Devilee P, (eds). World Health Organization Classification of Tumors Pathology and Genetics of Tumors of the Breast and Female Genital Organs. IARC Press: Lyon, 2003.

2 McDivitt RW, Stewart FW. Breast carcinoma in children. JAMA 1966;195:388-390.

3 Rosen PP, Cranor ML. Secretory carcinoma of the breast. Arch Pathol Lab Med 1991;115:141-144.
4 Oberman HA. Secretory carcinoma of the breast in adults. Am J Surg Pathol 1980;4:465-470.

5 Tognon C, Knezevich SR, Huntsman D, et al. Expression of the ETV6-NTRK3 gene fusion as a primary event in human SBC. Cancer Cell 2002;2:367-376.

6 Knezevich SR, McFadden DE, Tao W, et al. A novel ETV6-NTRK3 gene fusion in congenital fibrosarcoma. Nat Genet 1998;18:184-187.

7 Knezevich SR, Garnett MJ, Pysher TJ, et al. ETV6NTRK3 gene fusions and trisomy 11 establish a histogenetic link between mesoblastic nephroma and congenital fibrosarcoma. Cancer Res 1998;58: 5046-5048.

8 Lannon CL, Sorensen PH. ETV6-NTRK3: a chimeric protein tyrosine kinase with transformation activity in multiple cell lineages. Semin Cancer Biol 2005;15: 215-223.

9 Perou CM, Sørlie T, Eisen MB, et al. Molecular portraits of human breast tumours. Nature 2000;406:747-752.

10 Sørlie T, Perou CM, Tibshirani R, et al. Gene expression patterns of breast carcinomas distinguish tumor subclasses with clinical implications. Proc Natl Acad Sci USA 2001;98:10869-10874.

11 Nielsen TO, Hsu FD, Jensen K, et al. Immunohistochemical and clinical characterization of the basal-like subtype of invasive breast carcinoma. Clin Cancer Res 2004;10:5367-5374.

12 Carey LA, Perou C, Livasy CA. Race, breast cancer subtypes, and survival in the Carolina Breast Cancer Study. JAMA 2006;295:2492-2502.

13 Vincent-Salomon A, Mac Grogan G, Couturier J, et al. Calibration of immunohistochemistry for assessment of HER2 in breast cancer: results of the French multicentric GEFPICS study. Histopathology 2003;42:337-347.

14 Lamovec J, Bracko M. Secretory carcinoma of the breast: light microscopical, immunohistochemical and flow cytometric study. Mod Pathol 1994;7: 475-479.

15 Botta G, Fessia L, Ghiringhello B. Juvenile milk protein secreting carcinoma. Virchows Arch A Pathol Anat Histol 1982;395:145-152.

16 Jacquemier J, Padovani L, Rabayrol L, et al. Typical medullary breast carcinomas have a basal/myoepithelial phenotype. J Pathol 2005;207:260-268.

17 Reis-Filho JS, Milanezi F, Steele D, et al. Metaplastic breast carcinomas are basal-like tumours. Histopathology 2006;49:10-21.

18 Azoulay S, Laé M, Fréneaux P, et al. KIT is highly expressed in adenoid cystic carcinoma of the breast, a basal-like carcinoma associated with a favorable outcome. Mod Pathol 2005;18:1623-1631.

19 Bryan BB, Schnitt SJ, Collins LC. Ductal carcinoma in situ with basal-like phenotype: a possible precursor to invasive basal-like breast cancer. Mod Pathol 2006;19:617-621.

20 Makretsov N, He M, Hayes M, et al. A fluorescence in situ hybridization study of ETV6-NTRK3 fusion gene in secretory breast carcinoma. Genes Chromosomes Cancer 2004;40:152-157.

21 Arce C, Cortes-Padilla D, Huntsman DG, et al. Secretory carcinoma of the breast containing the ETV6-NTRK3 fusion gene in a male: case report and review of the literature. World J Surg Oncol $2005 ; 3: 35$ 
22 Herz H, Cooke B, Goldstein D. Metastatic secretory breast cancer. Non-responsiveness to chemotherapy: case report and review of the literature. Ann Oncol 2000;11:1343-1347.

23 Maitra A, Tavassoli FA, Albores-Saavedra J, et al. Molecular abnormalities associated with secretory carcinomas of the breast. Hum Pathol 1999;30: 1435-1440.

24 McCahon E, Sorensen PH, Davis JH, et al. Nonresectable congenital tumors with the ETV6-NTRK3 gene fusion are highly responsive to chemotherapy. Med Pediatr Oncol 2003;40:288-292.

$25 \mathrm{Li} \mathrm{Z}$, Tognon CE, Godinho FJ, et al. ETV6-NTRK3 fusion oncogene initiates breast cancer from committed mammary progenitors via activation of AP1 complex. Cancer Cell 2007;12:542-558. 